\title{
STRUCTURE THEORY FOR EQUATIONAL CLASSES GENERATED BY QUASI-PRIMAL ALGEBRAS
}

BY

\author{
ROBERT W. QUACKENBUSH( $\left.{ }^{1}\right)$
}

\begin{abstract}
Quasi-primal algebras (which include finite simple polyadic and cylindric algebras) were introduced by $\mathrm{A}$. S. Pixley. In this paper equational clasises generated by quas i-primal algebras are investigated with respect to the following concepts : the congruence extension property, the amalgamation property and the amalgamation class, weak injectives and weak injective hulls, the standard semigroup of operators. A brief discussion of monadic algebras is included to illustrate the results of the paper.
\end{abstract}

1. Introduction. Quasi-primal algebras were introduced and studied by A.S. Pixley in [13], [14] and were further studied by the present author in [15]. This paper parallels two recent papers of Grätzer and Lakser ([7], [8]); most of their results for pseudocomplemented distributive lattices carry over to equational classes generated by quasi-primal algebras.

Let $\Re$ be quasi-primal (the definition and elementary properties are given in $\S 2$ ) and let $K(\$)$ be the equational class generated by $\$$. The major results of this paper are: $K(\Re)$ has the Congruence Extension Property. $K(\Re)$ has the Amalgamation Property if and only if it has enough injectives. In case $K(\Re)$ does not have the Amalgamation Property, the amalgamation class of $\mathcal{K}(\Re)$ is defined and a sufficient condition for an algebra to be in it is given; this condition is necessary if the algebra is finite but an infinite algebra in the amalgamation class is given which does not satisfy the condition. The standard semigroup of operators for $K(\Re)$ is described. The weak injectives in $K(\Re)$ are determined and it is shown that in $\mathcal{K}(\Re)$ weak injective hulls are unique up to isomorphism. Finally, the last section is a brief discussion of monadic algebras illustrating the results of the paper.

The author thanks Harry Lakser and George Grätzer for several helpful discussions and suggestions.

Notational conventions. The symbol $\$$ denotes a quasi-primal algebra; unless otherwise noted, $\mathcal{K}$ denotes an equational class, and $K(\mathscr{U})$ denotes the equational class generated by $\mathcal{O}$.

Received by the editors June 28, 1972.

AMS (MOS) subject classifications (1970). Primary 08A05; Secondary 08A15, 08A25, $02 \mathrm{~J} 15$.

(1) This research was supported by a grant from the National Research Council of Canada. 
2. Quasi-primal algebras. Let $\mathcal{U}$ be a nontrivial algebra. $\mathcal{S}(\mathfrak{U})$ denotes the set of subalgebras of $\mathscr{X}$ and $\mathscr{Y}(\mathscr{U})$ denotes the set of all isomorphisms between (not necessarily distinct) nontrivial subalgebras of $\mathcal{O}$. Let $f: A^{n} \rightarrow A$; we say $f$ preserves $\mathcal{S}(\mathscr{C})$ if $f\left(a_{1}, \ldots, a_{n}\right) \in\left[a_{1}, \cdots, a_{n}\right]$ (the subalgebra generated by $\left.\left\{a_{1}, \ldots, a_{n}\right\}\right)$ for all $a_{1}, \ldots, a_{n} \in A$ and $f$ preserves $g(\mathfrak{U})$ if $\phi\left(f\left(a_{1}, \ldots, a_{n}\right)\right)=$ $f\left(\phi\left(a_{1}\right), \cdots, \phi\left(a_{n}\right)\right)$ for all $a_{1}, \cdots, a_{n} \in A$ and all $\phi \in \mathcal{Y}(\mathbb{P})$ with domain $\left[a_{1}, \ldots, a_{n}\right]$. $\mathscr{X}$ is quasi-primal if every function on $A$ which preserves $S(\mathscr{U})$ and $\mathscr{G}(\mathfrak{U})$ is a polynomial of $\mathfrak{U}$.

$\mathcal{K}(\mathscr{C})$ has distributive and permutable congruences if every $\mathbb{B} \in \mathcal{K}(\mathscr{U})$ has a distributive congruence lattice and permutable congruences. The ternary discriminator function, $t(x, y, z)$, is defined by

$$
t(x, y, z)= \begin{cases}z & \text { if } x=y, \\ x & \text { if } x \neq y\end{cases}
$$

The normal transform, $n(x, y, u, v)$, is defined by

$$
n(x, y, u, v)= \begin{cases}u & \text { if } x=y, \\ v & \text { if } x \neq y .\end{cases}
$$

Theorem 2.1. Let थ be a finite nontrivial algebra; the following are equiv. alent:

1. Ut is quasi-primal.

2. $K(\mathfrak{U})$ bas distributive and permutable congruences and eacb subalgebra of OU is simple.

3. The ternary discriminator function is a polynomial of $\mathrm{U}$.

4. The normal transform is a polynomial of 2 .

Theorem 2.2. Every $\mathfrak{B} \in \mathcal{K}(\mathfrak{B})$ is isomorpbic to a subdirect product of subalgebras of $\mathfrak{B}_{;}$; if $\mathbb{B}$ is finite then it is isomorpbic to a direct product of subalgebras of $\$$.

For Theorem 2.1, $1 \leftrightarrow 2$ is Theorem 5.1 of [13]; $1 \leftrightarrow 3$ is Theorem 3.2 of [14]. For $3 \leftrightarrow 4$, the fact that each function is definable in terms of the other is due independently to T.-K. Hu and W. Lampe. Theorem 2.2 is Theorem 4.1 of [13].

Let $\mathcal{K}$ be a class of algebras, $\mathscr{X} \in \mathcal{K}$. $\mathscr{U}$ is injective in $\mathcal{K}$ if for any $\mathscr{U}_{1}, \mathscr{V}_{2}$ $\epsilon \mathcal{K}$, any homomorphism $b: \mathscr{U}_{1} \rightarrow \mathscr{U}$ and any monomorphism $g: \mathscr{U}_{1} \rightarrow \mathscr{U}_{2}$, there is a homomorphism $f: \mathscr{U}_{2} \rightarrow \mathscr{U}$ such that $f g=b$. The next result is Theorem 5.6 of [15].

Theorem 2.3. If every $\phi \in \mathcal{I}(\Re)$ can be extended to an automorpbism of $\Re$ then the injectives in $K(\Re)$ are isomorpbic to the Boolean extensions of $\$$ by 
complete Boolean algebras. If not every $\phi \in \mathscr{G}(\Re)$ can be extended to an automorphism of $\$$ then $K(\$)$ bas no nontrivial injectives.

3. The Congruence Extension Property. A class of algebras $K$ has the Congreunce Extension Property (C.E.P.) if for any $\mathcal{Q}, B \in \mathcal{K}$ with $\mathscr{U} \subseteq B$ and any congruence $\theta$ of $\mathcal{U}$ there is a congruence $\psi$ of $B$ such that $\left.\psi\right|_{A}=\theta$.

Let $\theta(a, b)$ be the smallest congruence making $a$ congruent to $b$.

Lemma 3.1. Let $\mathfrak{U} \subseteq \Pi_{i \in I} \mathfrak{U}_{i}$. Let $a, b, c, d \in A$ witb $c \equiv d(\theta(a, b))$ and $a_{j}=b_{j}$ for some $j \in I$; then $c_{j}=d_{j}$.

Proof. Let $\operatorname{ker}\left(\pi_{j}\right)$ be the kernel of the projection of $\mathcal{U}$ onto $\mathcal{U}_{j^{\bullet}}$ Then $a_{j}=$ $b_{j}$ implies $a \equiv b\left(\operatorname{ker}\left(\pi_{j}\right)\right)$. Thus $c \equiv d(\theta(a, b))$ implies $c \equiv d\left(\operatorname{ker}\left(\pi_{j}\right)\right)$ and so $c_{j}=d_{j}$

Let $N(x, y, u, v)$ be the polynomial of $\Re$ which represents the normal transform on $\$$.

Theorem 3.2. Let $\mathbb{B} \in \mathcal{K}(\Re)$. Let $a, b, c, d \in B$. Then $c \equiv d(\theta(a, b))$ iff $N(a, b, c, d)=d$.

Proof. Let $N(a, b, c, d)=d$; then $c=N(a, a, c, d) \equiv N(a, b, c, d)=d(\theta(a, b))$. Conversely let $c \equiv d(\theta(a, b))$ and let $\mathscr{Q} \subseteq \bigotimes^{I}$. We must show that for all $i \in I$, $N\left(a_{i}, b_{i}, c_{i}, d_{i}\right)=d_{i}$; i.e. that $a_{i}=b_{i}$ implies $c_{i}=d_{i}$. But this is just Lemma 3.1.

The next lemma is due to Day [4].

Lemma 3.3. $K$ bas the C.E.P. iff for every $\mathscr{Q} \in \mathcal{K}$ and $a, b, c, d \in A, c \equiv$ $d(\theta(a, b))$ bolds in 2 iff it bolds in $[a, b, c, d]$.

Theorem 3.4. K(ß) bas the C.E.P.

4. Boolean extensions. The structure theory for quasi-primal algebras is closely connected with the theory of Boolean extensions. Boolean extensions were introduced by Foster [ 5$]$ and an extensive discussion of them can be found in [6]. In this paper Boolean extensions will be defined in a slightly different manner than in [6].

Let $\mathcal{U}$ be a finite algebra with $A=\left\{a_{1}, \ldots, a_{n}\right\}$ and let $B$ be a Boolean algebra. Let $S$ be the set of prime filters of $B$; identify $b \in B$ with $\{P \in S \mid b \in P\}$. The extension of $\mathcal{U}$ by $B$ will be defined as a particular subalgebra of $\mathfrak{Q}^{S}$. If $f \in A^{S}$ then $f$ induces a partition on $S$ via $P_{1} \sim P_{2}$ iff $f\left(P_{1}\right)=f\left(P_{2}\right)$; since $A$ is finite this partition is finite.

Definition. $f \in A[B]$ if and only if each partition class of the partition of $S$ induced by $f$ is a member of $B$.

Easy calculations show that $\mathscr{U}[\mathscr{B}]$ is a subalgebra of $\mathscr{U}^{S}$ and that $\mathscr{U}[\mathscr{R}]$ is isomorphic to the extension of $\mathscr{U}$ by $B$ as defined in [6]. It should be pointed 
out that the definition of $\mathfrak{U}[\mathscr{B}]$ is independent of the operations of $\mathfrak{A}$.

As the referee has pointed out, another well-known representation of Boolean extensions is that $\mathfrak{X}[\mathfrak{B}]$ is the algebra of all continuous maps from $\Omega \mathbb{B}$ to $A$ (with operations defined pointwise) where $\Omega B$ is the Stone space of $B$ and $A$ has the discrete topology.

An algebra $\mathscr{U}^{\prime} \subseteq \mathfrak{U}^{I}$ is a normal subdirect power of $\mathscr{U}$ if $\mathfrak{U}^{\prime}$ contains the diagonal subalgebra of $\mathfrak{Q}^{I}$ and is closed under the normal transform acting componentwise. By Theorems 22.3 and 22.4 of [6] any Boolean extension of $\mathcal{U}$ is isomorphic to a normal subdirect power of $\mathfrak{A}$ and conversely. If $\mathcal{U}$ is quasi-primal then the normal transform is a polynomial of $\mathcal{U}$ so that the normal subdirect powers of $\mathcal{O}$ are just the diagonal subdirect powers of $\mathfrak{A}$.

Let $B$ be the category of all Boolean algebras with all homomorphisms as

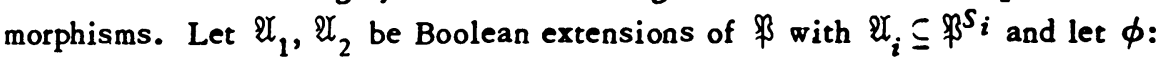
$\mathfrak{N}_{1} \rightarrow \mathfrak{X}_{2}$ be a homomorphism. Let $P=\left\{p_{1}, \ldots, p_{n}\right\}$ and let $\bar{P}=\left\{\bar{p}_{1}, \ldots, \bar{p}_{n}\right\}$ be the diagonal elements of $A_{1}$ (i.e. $\bar{p}_{i}(s)=p_{i}$ for all $s \in S_{1}$ ). Suppose there exist $i \neq j$ and $s \in S_{2}$ such that $\phi\left(\bar{p}_{i}\right)(s)=\phi\left(\bar{p}_{j}\right)(s)$, then since $n(x, y, u, v)$ is a polynomial of $\Re$ and $\phi()(s)$ is a homomorphism from $\mathcal{U}_{1}$ into $\Re, \phi(f)(s)=$ $\phi(g)(s)$ for all $f_{g} g \in A_{1}$. Thus let $T(\phi)=\left\{s \in S_{2} \mid \phi(f)(s)=\phi(g)(s)\right.$ for all $/ . g \epsilon$ $\left.A_{1}\right\}$. Call $\phi$ diagonal preserving if $T(\phi)=\varnothing$. Note that this includes the possibility that $S_{2}=\varnothing$ so that $\mathscr{U}_{2}$ is trivial. Hence we define $\Re[B]$ to be the category of all normal subdirect powers of $\$$ (i.e. Boolean extensions of $\$$ ) with all diagonal preserving homomorphisms as morphisms.

We now describe a functor $F: \mathfrak{B} \rightarrow \Re[B]$. For $\mathscr{B} \in \mathfrak{B}, F(\mathscr{B})=\Re[B]$. Let $\phi: \mathbb{B}_{1} \rightarrow \mathbb{B}_{2}$ and let $\mathbb{P}\left[\mathscr{B}_{i}\right] \subseteq \mathbb{P}^{S_{i}}$. Each $f \in P\left[B_{1}\right]$ can be described by an $n$-tuple, $\left(T_{1}, \cdots, T_{n}\right)$, of subsets of $S_{1}$ via $f(s)=a_{i}$ iff $s \in T_{i}$; a similar statement holds for $P\left[B_{2}\right]$. Then $F(\phi)(f)$ is the element of $A\left[B_{2}\right]$ corresponding to the $n$-tuple $\left(\phi\left(T_{1}\right), \cdots, \phi\left(T_{n}\right)\right)$. It is easy to check that $F$ is a functor and that $F(\phi)$ is $1-1$ (onto) iff $\phi$ is $1-1$ (onto).

Conversely let $\mathscr{A} \in \Re[B]$ with $\mathfrak{X} \subseteq \Re^{S}$. Let $\mathfrak{B}(\mathscr{Q})$ be the Boolean algebra of subsets $T \subseteq S$ such that there is an $f \in A$ and an $p \in P$ with $T=\{s \in S \mid f(s)=p\}$ (if $|A|=1$ then define $B(\mathscr{U})$ to be trivial). As in the proof of Theorem 22.4 of [6], $\mathfrak{R}(\mathfrak{U})$ is indeed a Boolean algebra; thus let $G(\mathfrak{U})=\mathscr{B}(\mathfrak{U})$. Now let $\mathfrak{U}_{1}, \mathscr{U}_{2} \epsilon$ $\Re[\Re]$ and let $\phi: \mathfrak{A}_{1} \rightarrow \mathfrak{U}_{2}$ be diagonal preserving. The case when $\mathscr{U}_{2}$ is trivial is obvious so assume $\mathscr{N}_{2}$ is not trivial. Let $\mathscr{N}_{i} \subseteq \beta^{S_{i}}$; let $\bar{P}=\left\{\bar{p}_{1}, \ldots, \bar{p}_{n}\right\}$ be the diagonal elements of $A_{1}$ and $\underline{P}=\left\{\underline{p}_{1}, \ldots, \underline{p}_{n}\right\}$ the diagonal elements of $A_{2}$.

Suppose that $\phi\left(F_{i}\right)=e_{i}$ for $1 \leq i \leq n$. Then for any $T_{1}$ in $B\left(\mathscr{Q}_{1}\right)$ and any $T_{2}$ in $B\left(\mathscr{T}_{2}\right)$ define $G(\phi)\left(T_{1}\right)=T_{2}$ if and only if there are an $f \in A_{1}$ and a $p \in P$ such that $T_{1}=\left\{s \in S_{1} \mid f(s)=p\right\}$ and $T_{2}=\left\{s \in S_{2} \mid \phi(f)(s)=p\right\}$. Again a straightforward computation shows that $G(\phi)$ is a homomorphism from $B\left(\mathscr{R}_{1}\right)$ to $B\left(\mathscr{R}_{2}\right)$. 
Now suppose that there is an $i$ such that $\phi\left(\bar{\phi}_{i}\right) \neq \ell_{i}$. Let $s \in S_{2}$; the mapping $p \rightarrow \phi(\bar{p})(s)$ is an automorphism of $\Re$. Thus let $\psi_{1}, \cdots, \psi_{k}$ be the distinct automorphisms of $\Re$ which are induced by elements of $S_{2}$ and let $\left\{T_{1}, \ldots, T_{k}\right\}$ be the partition of $S_{2}$ such that $s \in T_{i}$ iff $s$ induces $\psi_{i}$. It is easily seen that each $T_{i}$ is in $\mathfrak{B}\left(\mathfrak{A}_{2}\right)$. Define $\psi: \mathfrak{A}_{2} \rightarrow \mathfrak{U}_{2}$ as follows: for any $f \in A_{2}$ and any $s \in S_{2}, \psi(f)(s)=\psi_{i}^{-1}(f(s))$ iff $s \in T_{i}$. Since each $T_{i}$ is contained in $B\left(\mathscr{P}_{2}\right), \psi$ is an automorphism of $\mathfrak{U}_{2}$. Thus $\psi \phi\left(\bar{p}_{i}\right)=p_{i}$ for $1 \leq i \leq n$. Hence define $G(\phi)=$ $G(\psi \phi)$. In particular, $G(\psi)$ is the identity mapping on $\mathscr{E}\left(\mathfrak{U}_{2}\right)$. Clearly $G$ is a functor and $G(\phi)$ is $1-1$ (onto) if and only if $\phi$ is $1-1$ (onto).

Lemma 4.1. Let $\mathfrak{B}_{1}, \mathfrak{B}_{2}$ be nontrivial Boolean algebras. $\mathfrak{B}\left[\mathfrak{B}_{1}\right]$ is isomorphic to a subalgebra of $\Re\left[B_{2}\right]$ iff $B_{1}$ is isomorpbic to a subalgebra of $\mathscr{B}_{2} ; \Re\left[\mathscr{B}_{1}\right]$ is a bomomorphic image of $\Re_{2}\left[B_{2}\right]$ iff $B_{1}$ is a bomomorphic image of $\mathbb{B}_{2}$.

Proof. We need only comment on the case when $\phi$ is an embedding of $\$\left[\Re_{1}\right]$

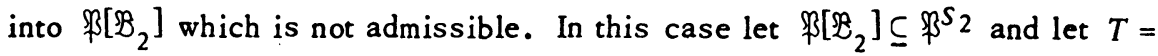
$\left\{s \in S_{2} \mid \phi(f)(s)=\phi(g)(s)\right.$ for all $\left.f, g \in P\left[B_{1}\right]\right\}$. Thus $T \neq \varnothing$; since $B_{1}$ is nontrivial, $T \nRightarrow S_{2}$. Thus choose $s_{0} \in S_{2}-T$ and define $\psi$ as follows: for any $f \in P\left[B_{1}\right]$ and any $s \in S_{2}$,

$$
\psi(f)(s)= \begin{cases}\phi(f)(s) & \text { if } s \notin T \\ \phi(f)\left(s_{0}\right) & \text { if } s \in T .\end{cases}
$$

Then $\psi$ is an admissible embedding of $\Re\left[\mathscr{B}_{1}\right]$ into $\Re\left[\mathscr{B}_{2}\right]$. Now the lemma is obvious.

Let $\mathfrak{X} \in \mathbb{P}[\mathbb{R}]$ and let $\theta$ be a congruence on $\mathscr{X}$. Then $\theta$ induces a congruence $\bar{\theta}$ on $\mathscr{B}(\mathfrak{U})$ as follows: let $I=\left\{b \in \mathscr{B}(\mathscr{U}) \mid\right.$ there exist $f, g \in A^{\prime}$ such that $f \equiv g(\theta)$ and $f(s) \neq g(s)$ for all $s \in b\} ; l$ is an ideal of $\mathscr{B}(\mathscr{U})$ and $\vec{\theta}$ is the congruence induced by $I$. It is clear that $\mathfrak{U} / \theta \simeq \mathbb{B}[\mathbb{B}(\mathscr{U}) / \bar{\theta}]$.

Lemma 4.2. Let $\Re$ and $\Re^{*}$ be quasi-primal algebras based on the same set $P$; let $\mathfrak{X} \in \Re[\Re]$ and $\mathfrak{X}^{*} \in \Re^{*}[\Re]$ also have the same base set $A$. Then the con. gruence lattice of $\mathfrak{Q}$ is identical with the congruence lattice of $\mathfrak{U}^{*}$.

These two lemmas will be used in following sections.

A quasi-primal algebra $¥$ is primal if $¥$ has no proper subalgebras and no proper automorphisms. Hence if $\Re$ is primal, $\mathfrak{A}_{1}, \mathfrak{U}_{2} \in \Re[\mathfrak{B}]$ and $\phi: \mathfrak{U}_{1} \rightarrow \mathfrak{U}_{2}$ is a homomorphism then $\phi$ is admissible. Since every $\mathscr{A} \in \mathcal{K}(\Re)$ is isomorphic to a Boolean extension of $¥$ we can extend the functor $G$ to $K(\Re)$ by describing a Boolean algebra naturally associated with $\mathscr{U}$. Let $S$ be the set of all homomorphisms from $\mathscr{U}$ to $\Re$. Then $G(\mathscr{U})$ is the Boolean algebra of all subsets $T$ of $S$ such that $T \in G(\mathscr{U})$ iff there is an $a \in A$ and a $p \in P$ with $T=\{\pi \in S \mid \pi(a)=p\}$. By defining 
$G$ on homomorphisms in the obvious way we get the well-known result that $K(\$)$ and $B$ are equivalent as categories (see [10]).

5. The standard semigroup of operators. Let $\mathcal{K}$ be an equational class of algebras; for any class $K^{\prime} \subseteq \mathcal{K}, H\left(K^{\prime}\right), S\left(K^{\prime}\right)$, and $P\left(K^{\prime}\right)$ denote, respectively, the class of all homomorphic images, isomorphs of subalgebras, and isomorphs of direct products, of algebras in $K^{\prime}$. Let $\Sigma$ be the free monoid on $\{H, S, P\}$ with 0 as identity. For $\mathcal{K}^{\prime} \subseteq \mathcal{K}$ define $0\left(\mathcal{K}^{\prime}\right)=\mathcal{K}^{\prime}$; for $U, V \in \Sigma$ define $U V\left(\mathcal{K}^{\prime}\right)=$ $U\left(V\left(\mathcal{K}^{\prime}\right)\right)$. The standard semigroup of operators of $\mathcal{K}$ is the quotient of $\Sigma$ determined by setting $U=V$ for $U, V \in \Sigma$ whenever $U\left(\mathcal{K}^{\prime}\right)=V\left(\mathcal{K}^{\prime}\right)$ for all $\mathcal{K}^{\prime} \subseteq \mathcal{K}$. An ordering can be defined on the standard semigroup by setting $U \leq V$ whenever $U\left(\mathcal{K}^{\prime}\right) \subseteq V\left(\mathcal{K}^{\prime}\right)$ for all $\mathcal{K}^{\prime} \subseteq \mathcal{K}$. Pigozzi [12] has shown that for any equational class $K$, the standard semigroup is a quotient of the ordered monoid shown in Figure 1. We will show that the standard semigroup for $\mathcal{K}(\Re)$ is that of Figure 2 or Figure 3.

We first establish that the standard semigroup for $\mathcal{K}(\$)$ has the monoid of Figure 3 as a homomorphic image. To do this we need to use the theory of Boolean extensions as discussed in $\$ 4$.

Let $\Re^{\prime}$ be a minimal nontrivial subalgebra of $\Re$. Since $\mathcal{K}\left(\Re^{\prime}\right) \subseteq \mathcal{K}(\Re)$ it is clear that the standard semigroup for $K\left(\Re^{\prime}\right)$ is a homomorphic image of that for $K(\Re)$. We will show that the standard semigroup for $\Re^{\prime}$ is given by Figure 3. To do this we will make use of a result of Comer and Johnson [3] which shows that the standard semigroup for Boolean algebras is given by Figure 3; then we apply Lemma 4.1 and the result follows. But to apply Lemma 4.1 we need to deal with nontrivial Boolean algebras. For instance, to show that $H \underline{S}$ for Boolean algebras, it is sufficient to take $K^{\prime}=\left\{B^{*}\right\}$ where $B^{*}$ is a two element Boolean algebra; then $H\left(\left\{B^{*}\right\}\right)$ contains a trivial algebra but $S\left(\left\{B^{*}\right\}\right)$ does not. However, $\Re^{\prime} \simeq \Re^{\prime}\left[\Re^{*}\right]$ may have a trivial subalgebra.

Thus we must show that we need only nontrivial Boolean algebras to prove the result of Comer and Johnson. Let $B_{0}$ be a complete atomic Boolean algebra with countably many atoms and let $B_{1}$ be the finite-cofinite subalgebra of $\mathbb{B}_{0}$ (i.e. all finite joins of atoms and their complements). Note that $B_{1}$ is not a homomorphic image of $B_{0}$. Let $B_{2}$ be a free Boolean algebra on $\kappa_{1}$ free generators and let $B_{3}$ be a Boolean algebra generated by a chain of length $K_{1}$. Note that $B_{3}$ is a homomorphic image of $B_{2}$ but not a subalgebra of $B_{2}$ since no free Boolean algebra contains an uncountable chain. Using these Boolean algebras and some other obvious examples, the result of Comer and Johnson is seen to hold. For instance, to show that $H \not L P S$ consider $\left\{B_{2}\right\}$. Then $B_{3} \in H\left(\left\{B_{2}\right\}\right)$. To see that $B_{3} \notin P S\left(\left\{B_{2}\right\}\right)$ note that $B_{3}$ has an $x_{1}$-chain while all chains in $S\left(\left\{B_{2}\right\}\right)$ are countable. Thus if $\mathfrak{B}_{3} \in P S\left(\left\{\mathfrak{B}_{2}\right\}\right)$ it must be the product of $\kappa_{1}$ subalgebras of 
$B_{2}$ and so has cardinality at least $2^{K_{1}}$. But $\Re_{3}$ is generated by an $\boldsymbol{K}_{1}$-chain and so has cardinality only $\boldsymbol{K}_{1}$.

Theorem 5.2. Let $\$$ bave no proper nontrivial subalgebras; then the standard semigroup of $K(\Re)$ is given by Figure 3.

Proof. By the above discussion it is clear that the standard semigroup for $K(\Re)$ has the monoid of Figure 3 as a homomorphic image. Thus it remains to show that $S H=H S$ and $S P=H S P$. That $S H=H S$ follows from the fact that $K(\Re)$ has the C.E.P. That $S P=H S P$ follows from the fact that, for any nontrivial $B \in$ $K(\Re), S P(B)=K(\Re)$. This is easily proven using Theorem 2.2 and the fact that $\Re$ has no proper nontrivial subalgebras.

Theorem 5.3. Let $\Re^{\prime}$ be a nontrivial proper subalgebra of $\$$ sucb that $\Re^{\prime}$ contains no trivial subalgebra. Then the standard semigroup for $K(\beta)$ is given by Figure 2.

Proof. We need only show $S P \neq S P H=H S P$. That $S P H=H S P$ is clear from Theorem 2.2. To show that $S P \notin S P H$ we need only show $¥ \notin\left\{S P\left(\left\{\Re \times \Re^{\prime}\right\}\right)\right.$. But if $\Re \in S P\left(\left\{\Re \times \Re^{\prime}\right\}\right)$ then there is a homomorphism from $¥$ into $\Re^{\prime}$. Since $\$$ is simple, $\Re^{\prime} \neq \Re$, and $\Re^{\prime}$ contains no trivial subalgebra, that is impossible.

Theorem 5.4. Let every proper subalgebra of $\$$ contain a trivial subalgebra. Then the standard semigroup for $K(\Re)$ is given by Figure 3.

Proof. We need only show that $S P=H S P$. Let $K^{\prime} \subseteq K(\Re)$. Each $\mathscr{B} \in \mathcal{K}^{\prime}$ is isomorphic to a subdirect product of subalgebras of $\aleph_{\text {. Th }}$. Thus let $\bigotimes_{1}, \ldots, \Re_{m}$ be all subalgebras of $\mathbb{B}$ which appear as subdirect factors for some $\mathbb{B} \in \mathcal{K}^{\prime}$. Then $H S P\left(K^{\prime}\right)=H S P\left(\left\{\Re_{1}, \ldots, \Re_{m}\right\}\right)=S P\left(\left\{\Re_{1}, \ldots, \Re_{m}\right\}\right)$. Since $\operatorname{SP}\left(\mathcal{K}^{\prime}\right) \subseteq H S P\left(K^{\prime}\right)$, we need only show $\left\{\mathbb{B}_{1}, \ldots, \mathbb{P}_{m}\right\} \subseteq S P\left(\mathcal{K}^{\prime}\right)$. Let $\Re_{i}$ be a subdirect factor of $B \in \mathcal{K}^{\prime}$; let $\rho$ be a homomorphism from $B$ onto $\mathbb{P}_{i}$. For each $p \in P_{i}$ choose $b \in B$ such

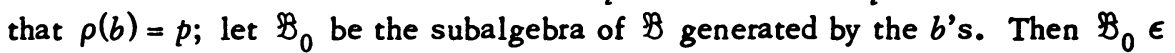
$S P\left(K^{\prime}\right)$ and $B_{0}$ is finite and so is a direct product of subalgebras of $\Re_{\text {. Since }}$ the restriction of $\rho$ to $B_{0}$ maps onto $\mathbb{B}_{i}$, one of the factors of $\mathbb{B}_{0}$ is $\mathbb{B}_{i}$. Since

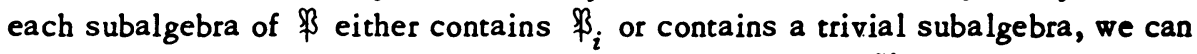
find a subalgebra of $\mathfrak{B}_{0}$ isomorphic to $\Re_{i}$. Hence $\Re_{i} \in S P\left(\mathcal{K}^{\prime}\right)$.

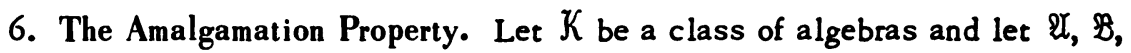

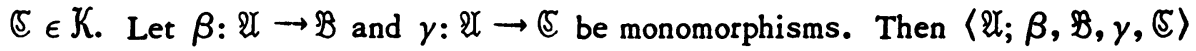
is an amalgam in $\mathcal{K}$. An amalgam $\langle\mathscr{N} ; \beta, \mathfrak{B}, \gamma, \mathfrak{E}\rangle$ can be amalgamated in $\mathcal{K}$ if there exist $\mathscr{D} \in \mathcal{K}$ and monomorphisms $\delta: \mathbb{B} \rightarrow \mathcal{D}$ and $\lambda: \mathbb{S} \rightarrow \mathcal{D}$ such that $\delta \beta=$ $\lambda y$. $\mathcal{K}$ has the Amalgamation Property (A.P.) if every amalgam in $\mathcal{K}$ can be amalgamated in $\mathcal{K}$. 
An extension $\sqrt{S}$ of an algebra $B$ is essential if for any congruence $\theta$ of $\mathfrak{E}$, $\left.\theta\right|_{B}=\omega_{B}$ implies $\theta=\omega_{C}$; i.e. if $c, d \in C$ with $c \neq 1 d$ and $c \equiv d(\theta)$ then there exist $a, b \in B$ with $a \neq 1 b$ such that $a \equiv b\left(\left.\theta\right|_{B}\right)$.

If $B \in \mathcal{K}(\Re)$ then $B \subseteq \bigotimes^{I}$ for some index set $I$. But then every essential extension of $\mathscr{B}$ in $K(\Re)$ is isomorphic to a subalgebra of $\Re^{I}$. Thus, up to isomorphism, every algebra in $K(\Re)$ has only a set of essential extensions. The following lemma is due to Banaschewski [1].

Lemma 6.1. Let $K$ be an equational class of algebras. Every algebra in $K$ can be embedded in an injective algebra in $\mathcal{K}$ iff every algebra in $\mathcal{K}$ bas only a set of essential extensions and $\mathcal{K}$ bas the C.E.P. and the A.P.

Theorem 6.2. $K(\Re)$ bas the A.P. iff every $\phi \in I(\Re)$ can be extended to an automorphism of $\Re$.

Proof. Apply Lemma 6.1 using Theorem 2.3, Theorem 3.4, and the fact that every $\mathfrak{B} \in \mathcal{K}(\mathbb{P})$ has only a set of essential extensions.

This result can be obtained in another way. Note first that the condition is obviously necessary. To show sufficiency we resort to Theorem 3 of [7].

Lemma 6.3. Let $K$ be an equational class of algebras with the C.E.P., and let every subalgebra of a subdirectly irreducible algebra in $K$ be subdirectly irreducible. Then $\mathcal{K}$ bas the A.P. iff every amalgam $\langle\mathfrak{X} ; \beta, \mathfrak{B}, \gamma, \mathbb{S}\rangle$ in $\mathcal{K}$ such that $\mathfrak{A}, \mathbb{B}, \mathcal{E}$ are subdirectly irreducible can be amalgamated in $\mathcal{K}$.

Corollary 6.4. If $\mathcal{K}$ is an equational class with the C.E.P. such that every subdirectly irreducible is simple and every subalgebra of a simple algebra is simple, then $\mathcal{K}$ bas the A.P. iff every amalgam $\langle\mathscr{X} ; \beta, \mathbb{B}, \gamma, \mathfrak{S}\rangle$ in $\mathcal{K}$ where $\mathscr{A}, \mathbb{B}, \mathfrak{E}$ are simple and $\mathscr{N}$ is nontrivial can be amalgamated into a simple algebra $\mathfrak{D}$ in $K$.

Proof. If $\mathcal{U}$ is trivial then the amalgam can be done in $B \times \mathfrak{C}$. Otherwise, by Lemma 6.3 we can amalgamate to some $\mathscr{D}$ in $\mathcal{K}$ which is not necessarily simple. Since $\mathscr{U}$ is nontrivial and is embedded in $\mathscr{D}$ and $\mathscr{U}, \mathfrak{B}, \mathcal{C}$ are simple, the amalgamation can be done in some simple factor of $\mathfrak{D}$.

Theorem 6.2 now follows since every simple algebra in $\mathcal{K}(\Re)$ can be embedded in $\$$ so that we need only consider amalgams of the form $\left\langle\Re^{\prime} ; \beta, \Re, \gamma, \Re\right\rangle$ where $\Re^{\prime} \subseteq \Re$.

Let Amal $(K)$, the amalgamation class of $K$, be all algebras $\mathscr{U} \in \mathcal{K}$ such that any amalgam $\langle\mathfrak{U} ; \beta, \mathfrak{E}, \gamma, \mathfrak{S}\rangle$ in $\mathcal{K}$ can be amalgamated in $\mathcal{K}$. The next lemma is proved in the same manner as Lemma 5 of [7]:

Lemma 6.5. $B \in A m a l(K(\Re))$ iff for any extension $\mathbb{S}$ of $B$ in $K(\Re)$ and any

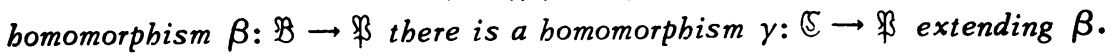


Theorem 6.6. Let $\Re_{1}, \cdots, \Re_{m}$ be all nontrivial subalgebras $\Re^{\prime}$ of $\aleph$ sucb that there is a $\phi \in \mathscr{I}(\Re)$ whose domain is $\Re^{\prime}$ and which cannot be extended to an

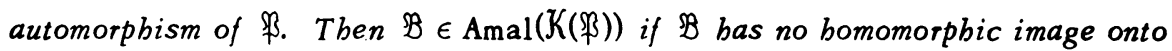
$\Re_{i}$ for $i=1, \ldots, m$.

Proof. Let $\rho: B \rightarrow \sharp$ be a homomorphism into $\Re$ and let $B \subseteq B^{\prime}$. Let $\theta=$ ker $\rho$ and let $\rho=a_{1} \pi_{1}$ where $\pi_{1}: B \rightarrow B / \theta$ and $\alpha_{1}: B / \theta \rightarrow \Re$ are canonical. By the C.E.P. there is a congruence $\theta^{\prime}$ of $B^{\prime}$ such that $\left.\theta^{\prime}\right|_{B}=\theta$; we may assume that $\theta^{\prime}$ is maximal with respect to this property so that $B^{\prime} / \theta^{\prime}$ is isomorphic to a subalgebra of $\Re$. Thus let $\alpha_{2}$ be an embedding of $\mathscr{R}^{\prime} / \theta^{\prime}$ into $\Re$; let $\pi_{2}$ : $B^{\prime} \rightarrow B^{\prime} / \theta^{\prime}$ be canonical and let $\iota$ be the embedding of $B / \theta$ in $B^{\prime} / \theta^{\prime}$ such that $\left.\pi_{2}\right|_{B}=\imath \pi_{1}$. Then $\alpha_{2} \iota \alpha_{1}^{-1} \in g(\Re)$ and so by assumption can be extended to an automorphism $\beta$ of $\Re$. But then $\beta^{-1} \alpha_{2} \pi_{2}$ extends $\rho$. Thus by Lemma $6.5, B \in$ $\operatorname{Amal}(K(\Re))$.

Theorem 6.7. If $B \in \operatorname{Amal}(K(B))$ is finite then $B$ satisfies the condition of Theorem 6.6.

Proof. Since $B$ is finite it is a direct product of subalgebras of $\$$ (see 2.2) and the only homomorphisms into $\$$ are the projections onto its simple factors followed by a $\phi \in I(\Re)$. Assume all simple factors are nontrivial and that there are $M$ of them; let $\pi_{i}: \mathfrak{R} \rightarrow \Re_{i}$ be the projection onto the $i$ th factor and let $\phi \epsilon$ $g(\Re)$ have domain $\mathfrak{F}_{i}$. We must show that $\phi$ can be extended to an automorphism of $\Re$. But $B \subseteq \Re^{M}$ and $B \in A \operatorname{mal}(K(\Re))$ says that $\phi \pi_{i}$ can be extended to $\rho$ on $\Re^{M}$. Clearly $\rho$ is the $i$ th projection on $\Re^{M}$ followed by an automorphism $\alpha$ of $\aleph_{\text {. }}$ It is obvious that $\alpha$ extends $\phi$.

7. Weak injectives. Let $K$ be a class of algebras and $\mathscr{U} \in \mathcal{K}$. $\mathscr{U}$ is a weak injective of $\mathcal{K}$, if, for any $\mathfrak{X}_{1}, \mathfrak{X}_{2} \in \mathcal{K}$, any homomorphism $b: \mathfrak{X}_{1} \rightarrow \mathfrak{U}$ onto $\mathfrak{A}$ and any monomorphism $g: \mathfrak{A}_{1} \rightarrow \mathfrak{U}_{2}$ there is a homomorphism $f: \mathfrak{U}_{2} \rightarrow \mathfrak{U}$ such that $f g=b$. $\mathscr{A}$ is a retreat of $\mathbb{B}$ if there is a monomorphism $g: \mathfrak{A} \rightarrow \mathfrak{B}$ and an epimorphism $f: \mathbb{B} \rightarrow \mathfrak{U}$ such that $f g$ is the identity on $\mathfrak{U}$. $\mathscr{U}$ is an absolute subre. tract in $\mathcal{K}$ if it is a retract of every extension in $\mathcal{K}$. These concepts are tied together by Lemma 7.1 which is a combination of Lemmas $1-3$ of [8].

Lemma 7.1. Let $K$ be an equational class of algebras. If $\mathfrak{A}$ is injective in $\mathcal{K}$ then it is a weak injective in $\mathcal{K}$; if $\mathfrak{A}$ is a weak injective in $\mathcal{K}$ then it is an absolute subretract in $K$. If $K$ bas the C.E.P. and $\mathcal{X}$ is an absolute subretract in $K$ then $\mathcal{X}$ is a weak injective in $K$. If $K$ bas the C.E.P. and the A.P. and $\mathscr{U}$ is an absolute subretract in $K$ then $\mathscr{A}$ is injective in $K$.

Recall that $K(\Re)$ has the C.E.P.; thus in $K(\Re)$ absolute subretracts and weak injectives coincide. In general, $K(\$)$ does not have the A.P. and so has no non- 
trivial injectives; thus it is natural to look at weak injectives in $K(\$)$. A maximal subdirectly irreducible algebra in an equational class $K$ is a subdirectly irreducible algebra such that no proper extension in $K$ is subdirectly reducible. The next result is Lemma 4 of [8].

Lemma 7.2. Let $K$ be an equational class of algebras. Then any maximal subdirectly irreducible algebra in $\mathrm{K}$ is an absolute subretract in $\mathrm{K}$.

Theorem 7.3. $\$$ is a weak injective in $K(\$)$ but no proper nontrivial subalgebra of $¥$ is a weak injective in $K(\$)$.

Proof. That $\$$ is a weak injective in $K(\Re)$ follows from Lemmas 7.1 and 7.2. That a proper nontrivial subalgebra $\Re^{\prime}$ of $\$$ is not a weak injective in $K(\Re)$

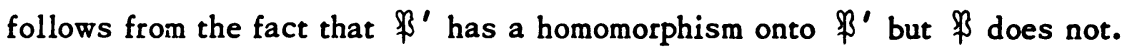

It is clear that a direct product of weak injectives is a weak injective. However, with regard to retracts of weak injectives, we must proceed more carefully. Let $g$ be an embedding of $B$ as a subdirect product of $\left\{B_{i} \mid i \in I\right\}$. Then $B$ is a subdirect retract of $\left\{\mathscr{B}_{i} \mid i \in I\right\}$ if there is a homomorphism $f$ from $\Pi_{i \in I} B_{i}$ onto $B$ such that $f g$ is the identity on $B$. The next result is Lemma 5 of [8].

Lemma 7.4. A subdirect retract of a family of weak injectives is a weak injective.

Theorem 7.5. The weak injectives in $K(\$)$ are precisely the Boolean extensions of $¥$ by complete Boolean algebras.

Proof. The proof is similar to the proof of Theorem 5.6 of [15] and will only be sketched. Expand the type of $¥$ so that each element of $¥$ is the value of a nullary operation; call the new algebra $\Re^{*}$. Then $\beta^{*}$ is primal so that the weak injectives in $K\left(\Re^{*}\right)$ are the Boolean extensions of $\beta^{*}$ by complete Boolean algebras. Each is a subdirect retract of some direct power of $\Re^{*}$; thus by ignoring the added nullaries, each is a weak injective in $K(\Re)$. Conversely let $\mathcal{W}$ be a weak injective in $K(\Re)$. Then $B$ can be embedded in $\Re^{I}$ for some $I$; since $B$ is

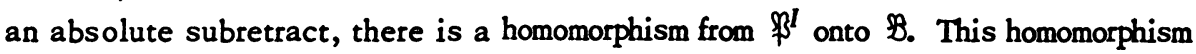
interprets the added nullaries of $\sharp^{*}$ in $B$ (via the diagonal subalgebra of $\wp^{I}$ ); let $B^{*}$ denote $B$ with the added nullaries. Then $B^{*}$ is a homomorphic image of $\left(B^{*}\right)^{I}$ and so $B^{*} \in K\left(B^{*}\right)$. Since $B$ is a weak injective in $K\left(B_{)}\right), B^{*}$ is a weak

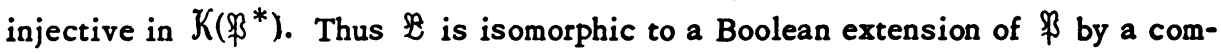
plete Boolean algebra.

8. Weak injective hulls.

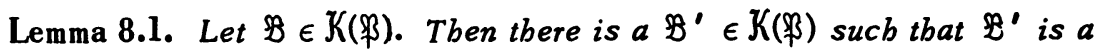
maximal essential extension of $B$. Furthermore, eacb sucb $B^{\prime}$ is a weak injective in $K(\Re)$. 
Proof. As remarked in $\S 5$, each $B \in K(\Re)$ has only a set of essential extensions in $K(\Re)$ so that an application of Zom's lemma shows that $B$ has a maximal essential extension $B^{\prime}$ in $K(\mathbb{P})$. Clearly a maximal essential extension is an absolute subretract; since $K(\Re)$ has the C.E.P., $B^{\prime}$ is a weak injective in K( $($ ) .

Thus any maximal essential extension of $\mathscr{B}$ in $K(\Re)$ will be called a weak injective hull of $B$. It is well known that injective hulls are unique up to isomorphism. The following example shows that a similar result does not hold for weak injective hulls.

Example 8.2. Let $¥$ have proper nontrivial subalgebras $\bigotimes_{1}, \Re_{2}, \bigotimes_{3}$ such that neither $\aleph_{1}$ nor $\sharp_{2}$ is embeddable in the other and $\sharp_{1} \cap \Re_{2}=\Re_{3}$. Consider $K\left(\Re_{1} \times \Re_{2}\right)$. Then $\Re_{1}$ and $\Re_{2}$ are maximal subdirectly irreducible algebras in $K\left(\Re_{1} \times \Re_{2}\right)$ and so, by Lemma 7.2, are absolute subretracts in $K\left(\Re_{1} \times \Re_{2}\right)$. Since $K(\Re)$ has the C.E.P. so does $K\left(\Re_{1} \times \Re_{2}\right)$. Thus $\Re_{1}$ and $\oiint_{2}$ are weak injectives in $K\left(\oiint_{1} \times \oiint_{2}\right)$. Clearly they are maximal essential extensions of $\oiint_{3}$ and so $\bigotimes_{1}$

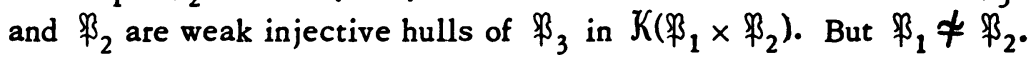

This negative result is countered by the following positive results.

Theorem 8.3. Let $\mathcal{U} \in A \mathrm{mal}(\mathcal{K})$ and let $B_{1}$ and $B_{2}$ be two weak injective bulls of $\mathscr{N}$; then $B_{1} \simeq \mathfrak{B}_{2}$.

Proof. Let $\beta: \mathscr{U} \rightarrow \mathbb{B}_{1}$ and $\gamma: \mathfrak{U} \rightarrow \mathbb{B}_{2}$ be embeddings; then $\left\langle\mathscr{U} ; \beta, \mathbb{B}, \gamma, \mathbb{B}_{2}\right.$ 〉 is an amalgam in $\mathcal{K}$. Since $\mathscr{U} \in \operatorname{Amal}(\mathcal{K})$ it can be amalgamated to $\mathbb{E} \in \mathcal{K}$. Since $B_{2} \subseteq \mathbb{C}$ there is a retraction $\rho$ of $C$ onto $B_{2}$. Since $B \subseteq B_{2}, \rho$ is $1-1$ on $B$. Since $B \subseteq B_{1}$ and $B_{1}$ is an essential extension of $B$, the restriction $\bar{\rho}$ of $\rho$ to $B_{1}$ must be $1-1$. But $B_{1}$ is a maximal essential extension of $B$ and so $\bar{\rho}$ must map onto $B_{2}$; thus $\mathfrak{B}_{1} \simeq \mathfrak{B}_{2}$.

Theorem 8.4. Weak injective bulls in $K(\$)$ are unique up to isomorphism.

Proof. If $\Re=\langle P ; F\rangle$ let $\Re^{*}=\langle P ; n(x, y, u, v)\rangle(n(x, y, u, v)$ is the normal transform). Thus $\Re^{*}$ is quasi-primal and $g\left(\Re^{*}\right)$ consists of all bijections between subsets of $P$ of at least two elements. Hence every $\phi \in I^{(}\left(\mathbb{P}^{*}\right)$ can be extended to an automorphism of $\beta^{*}$ so that weak injectives in $K\left(\beta^{*}\right)$ are injectives. Let $\Re\left[B_{1}\right]$ and $\Re\left[\Re_{2}\right]$ be isomorphic to weak injective hulls of $\mathcal{Q} \in K(\Re)$. Let $\Re^{*}=$ $\left[B_{1}\right], \Re^{*}\left[B_{2}\right]$ and $\mathscr{U}^{*}$ be the corresponding algebras in $K\left(\Re^{*}\right)$ based on the same sets as $\Re\left[\mathscr{B}_{1}\right], \Re\left[\mathscr{B}_{2}\right]$ and $\mathscr{U}$ (recall that $n(x, y, u, v)$ is necessarily a polynomial of $\Re$ ). By Theorem 7.5 and Lemma $4.2, \Re^{*}\left[\mathscr{B}_{1}\right]$ and $\Re^{*}\left[\Re_{2}\right]$ are isomorphic to weak injective hulls (and hence injective hulls) of $\mathfrak{B}_{1}^{*}$. Thus $\mathfrak{B}^{*}\left[\mathbb{B}_{1}\right] \simeq \Re^{*}\left[\mathbb{B}_{2}\right]$ so that $\mathscr{B}_{1} \simeq \mathfrak{B}_{2}$. But then $\mathfrak{Q}\left[\mathfrak{B}_{1}\right] \simeq \Re\left[\mathfrak{B}_{2}\right]$.

Problems. (1) If $\mathfrak{U}_{1}, \mathfrak{U}_{2}$ are extensions of $\mathfrak{U}$ which are injective hulls then 
the above proof does not guarantee the existence of an isomorphism $\phi: \mathscr{U}_{1} \rightarrow \mathscr{U}_{2}$ which is constant on $\mathfrak{U}$ (i.e. the isomorphism between $\mathfrak{U}_{1}^{*}$ and $\mathfrak{U}_{2}^{*}$ need not be an isomorphism between $\mathscr{U}_{1}$ and $\mathfrak{U}_{2}$ ). Does such a $\phi$ always exist?

(2) Find some general conditions which guarantee the uniqueness of weak injective hulls. In particular, if every $\mathscr{U}$ in $\mathcal{K}$ has a weak injective hull and each subdirectly irreducible of $\mathcal{K}$ has a unique weak injective hull then are weak injective hulls in $K$ unique?

9. The amalgamation class of $K(\Re)$. Let $\Re^{\prime} \subseteq \Re$ be nontrivial. Call $\Re^{\prime}$ good if every $\phi \in I(\Re)$ with domain $\beta^{\prime}$ can be extended to an automorphism of $\Re$; otherwise call $\Re^{\prime}$ bad. Theorems 6.6 and 6.7 state that if $\mathscr{A} \in \mathcal{K}(\Re)$ is finite then $थ \in A m a l(K(\Re))$ if and only if one of the two following equivalent conditions holds :

(*) $\mathcal{U}$ has no homomorphism onto a bad subalgebra of $¥$.

$(* *) \mathcal{U}$ is a subdirect product of good subalgebras of $\Re$.

In general $(* *)$ is weaker than $(*)$; Theorem 6.6 states that $(*)$ is sufficient for any $\mathfrak{U} \in \mathcal{K}(\Re)$ to be in $\operatorname{Amal}(\mathcal{K}(\Re))$. In this section we will show that $(* *)$ is not sufficient and $(*)$ is not necessary.

Example 9.1. Let $\oiint_{1} \subseteq \$$ be nontrivial; let $\phi \in g(\Re)$ have domain $\oiint_{1}$ and not be extendible to an automorphism of $\Re$. Let $I$ be a countable set; define $\mathscr{U}$ $\subseteq \Im^{I}$ as follows: $f \in A$ iff there is a $p \in P_{1}$ such that $f(i)=p$ a.e. (that is, for all but finitely many $i \in I$ ). It is easily seen that $\mathscr{X}$ is a subdirect power of $\$$ (see $\S 23$ of [6]). The relation $f \sim g$ iff $f=g$ a.e. is a congruence of $\mathscr{A}$; define the map $\rho: \mathfrak{U} \rightarrow \Re$ by $\rho(f)=\phi(p)$ iff $f(i)=p$ a.e. Clearly $\rho$ is a homomorphism. Suppose $\rho$ can be extended to a homomorphism $\bar{\rho}: \Re^{I} \rightarrow \mathfrak{\beta}$. Let $\bar{\beta}$ be the diagonal subalgebra of $\mathfrak{P}^{I}$; then $\left.\bar{\rho}\right|_{\bar{p}}$ could be used to extend $\phi$ to an automorphism of $\Re$ contrary to assumption. Thus no such $\bar{\rho}$ exists and hence $\mathfrak{U} \notin \operatorname{Amal}(\mathcal{K}(\mathfrak{P}))$. Since $\mathcal{Q}$ is a subdirect power of $¥$ and $¥$ is good, $(* *)$ is not sufficient.

Example 9.2. Let $P=\{0,1,2\}, P_{0}=\{0,1\}$. Define $u: P \rightarrow P$ by $u(0)=1$, $u(1)=0, u(2)=1$. Let $\beta=\langle P ; t(x, y, z), u(x)\rangle$ and let $\phi(0)=1, \phi(1)=0$. Then $\Re$ is quasi-primal, $\bigotimes_{0}$ is its only proper subalgebra, and $\phi$ is an automorphism of $\oiint_{0}$ which cannot be extended to an automorphism of $\oiint_{\text {. Let }} I_{0}$ and $I_{1}$ be countable sets with $I_{0} \cap I_{1}=\varnothing$ and $I_{0} \cup I_{I}=I$. Let $\overline{0}, \overline{1}, \in P^{I}$ be defined by $\overline{0}(i)=j$ for $i \in I_{j}$ and $\overline{1}(i)=j$ for $i \in I_{1-j}, j=0,1$. Let $f \in P^{I}$, then $f \in A^{\prime}$ iff $f=\overline{0}$ a.e. or $f=\overline{1}$ a.e. As in Example $9.1, \mathfrak{X}^{\prime}$ is a subdirect power of $\Re$. We will show that $\mathscr{U}^{\prime} \in \operatorname{Amal}(\mathcal{K}(\Re))$ so that condition $(*)$ is not necessary (note that $\mathcal{U}^{\prime}$ has $\sharp_{0}$ as a homomorphic image).

Lemma 9.3. Let $\mathfrak{U} \in \mathcal{K}(\mathfrak{\beta})$. Then $\mathfrak{U} \in \operatorname{Amal}(\mathcal{K}(\Re))$ iff for any bomomorphism $\rho: \mathfrak{U} \rightarrow \Re$ and any weak injective bull $\mathfrak{U}_{1}$ of $\mathfrak{U}$ there is a bomomorphism $\bar{\rho}: \mathfrak{U}_{1}$

$\rightarrow \Re$ which extends $\rho$. 
Proof. By Lemma 6.5 the condition is necessary. Let $\mathscr{A} \subseteq \mathscr{P}_{2}$. If $\mathscr{A}_{2}$ is an essential extension of $\mathscr{U}$ then let $\mathfrak{U}_{1}$ be a weak injective hull of $\mathscr{U}_{2}$ and hence of $\mathscr{U}$. Thus $\rho: \mathfrak{U} \rightarrow \mathfrak{W}$ can be extended to $\bar{\rho}: \mathfrak{U _ { 1 }} \rightarrow \mathfrak{B}$ and so $\left.\bar{\rho}\right|_{A_{2}}$ extends $\rho$ to $\mathscr{U}_{2}$. Otherwise by the corollary to Lemma 3 in [8] there is a homomorphism $\psi$ from $\mathfrak{U}_{2}$ to an essential extension of $\mathscr{U}$ which leaves $\mathscr{U}$ fixed. Thus $\rho$ can be extended to $\mathfrak{H}_{2}$ and $\mathfrak{U} \in \operatorname{Amal}(\mathcal{K}(\mathfrak{P}))$.

It is easy to see that $\mathfrak{P}^{I}$ is an essential extension of $\mathscr{U}^{\prime}$; thus $\mathfrak{P}^{I}$ is a weak injective hull of $\mathscr{Q}^{\prime}$ ' and so by Theorem 8.4 every weak injective hull of $\mathscr{Q}^{\prime}$ is isomorphic to $\$$ I'.

Let $\theta$ be a congruence on $\mathscr{X}^{\prime}$ such that $\mathscr{X}^{\prime} / \theta \simeq \Re_{0}$; clearly $\overline{0} \equiv \overline{\mathrm{I}}(\theta)$. Let $f=\overline{0}$ a.e. If $f \equiv \overline{1}(\theta)$ then let $J=\{i \in I \mid f(i)=\overline{1}(i)\}$; since $f=\overline{0}$ a.e., $J$ is finite. Let $\theta_{J}$ be the kernel of the projection of $\mathfrak{U}^{\prime}$ into $\Re^{J}$; clearly $\theta \geq \theta_{J^{\prime}}$. However, it is easily seen that $\mathscr{U} \% \theta_{J} \simeq \mathfrak{S}_{J}^{J}$ so that $\mathscr{Q} \% / \theta$ cannot be isomorphic to $\mathbb{P}_{0}$. Hence $f \equiv \overline{0}(\theta)$; similarly if $f=\overline{1}$ a.e. then $f \equiv \overline{1}(\theta)$. Thus $\theta$ is the only congruence of $\mathfrak{U}^{\prime}$ such that $\mathfrak{Q} \mathrm{U}^{\prime} / \theta \simeq \Re_{0}$.

Therefore there are exactly two homomorphisms $\psi_{1}, \psi_{2}$ from $2 \mathrm{U}^{\prime}$ to $\Re_{0} ; \psi_{1}$ is determined by $\psi_{1}(\overline{0})=0$ while $\psi_{2}$ is determined by $\psi_{2}(\overline{0})=1$. Let $\{\underline{0}, \underline{1}, \underline{2}\}$ be the diagonal subalgebra of $\Re^{I}$ and let $\psi$ be a homomorphism from $\mathfrak{P}^{I}$ to $\Re_{\text {; }}$; necessarily $\psi(\underline{0})=0, \psi(\underline{1})=1$ and $\psi(\underline{2})=2$. Let $\sigma$ be a congruence on $\mathfrak{P}^{I}$ and let it be associated with the filter $F(\sigma)$ of the power set of $l$. Then $\left.\sigma\right|_{A^{\prime}} \geq \theta$ if and only if $F(\sigma)$ contains every cofinite subset of $I$. Thus to extend $\psi_{1}$ take $\sigma$ so that $F(\sigma)$ is a nonprincipal ultrafilter containing $I_{0}$ while to extend $\psi_{1}$ take $F(\sigma)$ to be a nonprincipal ultrafilter containing $I_{1}$. On the other hand, if $\rho$ is a homomorphism of $\mathfrak{A l}^{\prime}$ onto $\mathfrak{P}$ then, using the C.E.P., $\rho$ can be extended to $\Re^{\prime}$. Thus every homomorphism from $\mathfrak{Q}^{\prime}$ to $\$$ can be extended to $\Re^{\prime}$.

Now consider the case where $\phi: \mathfrak{U}^{\prime} \rightarrow \Re^{I}$ is an embedding and $\Re^{I}$ is a weak injective hull of $\phi\left(\mathscr{U}^{\prime}\right)$. Let $I_{2}=\{i \in I \mid \phi(0)(i)=0$ and $\phi(1)(i)=1\}$ and $I_{3}=$ $\{i \in I \mid \phi(\overline{0})(i)=1$ and $\phi(\overline{1})(i)=0\}$. Since $\{0,1\}$ is the only proper subalgebra of $\Re$ and $\{\phi(0), \phi(\overline{1})\}$ is a subalgebra of $\phi\left(\mathscr{P} I^{\prime}\right), I_{2} \cap I_{3}=\varnothing$ and $I_{2} \cup I_{3}=I$.

We will show that there are bijections $\phi_{0}: I_{0} \rightarrow I_{2}$ and $\phi_{1}: I_{1} \rightarrow I_{3}$ such that for any $f \in A^{\prime}, \phi(f)\left(\phi_{j}(i)\right)=f(i)$ for $i \in I_{J}, j=0,1$. From this it is clear that 2' satisfies the condition of Lemma 9.3 so that $\mathfrak{U}^{\prime} \in \operatorname{Amal}(\mathcal{K}(\Re))$.

For $i \in I_{0}$ define $d_{i}, e_{i}, f_{i}, g_{i} \in A^{\prime}$ as follows: $d_{i}(j)=e_{i}(j)=\overline{1}(j)$ and $f_{i}(j)=$ $g_{i}(j)=\overline{0}(j)$ for $j \neq i$ and $d_{i}(i)=2, e_{i}(i)=0, f_{i}(i)=2, g_{i}(i)=1$. Note that $\left\{\overline{0}, \overline{1}, d_{i}, e_{i}, f_{i}, g_{i}\right\}$ forms a subalgebra of $\mathscr{U}^{\prime}$ and is generated by either $d_{i}$ or $f_{i}$.

Let $J(i)=\left\{j \in I \mid \phi\left(f_{i}\right)(j) \neq \phi(\overline{0})(j)\right\}$; clearly $J(i) \neq \varnothing$ for any $i \in I_{0}$. Let $j \epsilon$ $J(i)$. Noting that $u\left(f_{i}\right)=u(0)$, we see that $u\left(\phi\left(f_{i}\right)\right)(j)=u(\phi(0))(j)$ but $\phi\left(f_{i}\right)(j) \neq$ $\phi(0)(j)$. Hence $\phi\left(f_{i}\right)(j)=2$ and $\phi(0)(j)=0$ so that $J(i) \in I_{2}$. Let $F$ be a finite subset of $I_{0}$ and let $f_{F} \in A^{\prime}$ satisfy $f_{F}(i)=2$ for $i \in F$ and $f_{F}(i)=\overline{0}(i)$ other- 
wise. Then an easy induction shows that $\phi\left(f_{F}\right)(j)=2$ for $j \in \bigcup_{i \in F} J(i)$ and $\phi\left(f_{F}\right)(j)=\phi(\overline{0})(j)$ otherwise. For instance, if $F=\{i, j\}$ then $f_{F}=t\left(f_{i}, \overline{0}, f_{j}\right)$ so $\phi\left(f_{F}\right)=t\left(\phi\left(f_{i}\right), \phi(\overline{0}), \phi\left(F_{j}\right)\right)$.

Since $f_{i}=t\left(f_{i}, \overline{0}, g_{i}\right)$ and $g_{i}=t\left(g_{i}, \overline{0}, f_{i}\right)$ we have $\phi\left(f_{i}\right)=t\left(\phi\left(f_{i}\right), \phi(\overline{0}), \phi\left(g_{i}\right)\right)$ and $\phi\left(g_{i}\right)=t\left(\phi\left(g_{i}\right), \phi(0), \phi\left(f_{i}\right)\right)$. Hence $J(i)=\left\{j \in I \mid \phi\left(g_{i}\right)(j) \neq \phi(\overline{0})(j)\right\}$. By using $n(x, y, u, v)$ (note that $n(x, y, u, v)$ is a polynomial of $\$$ ) we get that $J(i)=$ $\left\{j \in I \mid \phi\left(d_{i}\right)(j) \neq \phi(\overline{1})(j)\right\}=\left\{j \in I \mid \phi\left(e_{i}\right)(j) \neq \phi(\overline{1})(j)\right\}$.

Since $\left\{\phi\left(f_{i}\right), \phi\left(g_{i}\right)\right\}$ must generate a 6 element subalgebra of $\phi\left(\mathscr{Z}{ }^{\prime}\right)$, a simple calculation shows that $\phi\left(g_{i}\right)(j)=1$ for all $j \in J(i)$. A similar analysis shows that $\phi\left(d_{i}\right)(j)=2$ and $\phi\left(e_{i}\right)(j)=0$ for $j \in J(i)$.

Now let $i, j \in I_{0}$ with $i \neq j$ and let $b \in A^{\prime}$ satisfy $b(i)=2, b(j)=1$ and $b(k)=$ $\overline{0}(k)$ otherwise. Since $b=t\left(f_{i}, \overline{0}, g_{j}\right)=t\left(g_{j}, \overline{0}, f_{i}\right)$ then $\phi(b)=t\left(\phi\left(f_{i}\right), \phi(\overline{0}), \phi\left(g_{j}\right)\right)$ $=t\left(\phi\left(g_{j}\right), \phi(\overline{0}), \phi\left(f_{i}\right)\right)$. Hence $J(i) \cap J(j)=\varnothing$.

For $i \in I_{1}$, define $d_{i}^{\prime}, e_{i}^{\prime}, f_{i}^{\prime}, g_{i}^{\prime}$ and $J^{\prime}(i)$ as for $i \in I_{0}$. Clearly the corresponding results will hold for $J^{\prime}(i)$. Thus for $i \in I_{0}$ let $\phi_{0}(i)=J_{(}(i)$ and for $i \in I_{1}$ let $\phi_{1}(i)=J^{\prime}(i)$. Then it is easily seen that for any $f \in A^{\prime}, \phi(f)(j)=f(i)$ if $j \epsilon$ $\phi_{0}(i)$ or if $j \in \phi_{1}(i)$. It now remains to be shown that $I_{2}=\bigcup_{i \in I_{0}} J(i)$ and $I_{3}=$ $\bigcup_{i \in I_{1}} J^{\prime}(i)$ and that $|J(i)|=1$ for $i \in I_{0}$ and $\left|J^{\prime}(i)\right|=1$ for $i \in I_{1}$.

Suppose $j \in I_{2}-\bigcup_{i \in I_{0}} J(i)$. Let $f \in A^{\prime}$; if $f=\overline{0}$ a.e. then $\phi(f)(j)=0$ while if $f=\overline{1}$ a.e. then $\phi(f)(j)=1$. Thus if $f, g \in A^{\prime}$ and $\phi(f)(i)=\phi(g)(i)$ for all $i \neq j$ then $\phi(f)=\phi(g)$. Let $\theta_{j}$ be the kernel of the projection of $\$^{I}$ onto $\mathbb{P}^{I-\{j\}}$; clearly $\theta_{j} \neq \omega$ while $\left.\theta_{j}\right|_{\phi\left(A^{\prime}\right)}=\omega$. This contradicts the assumption that $\Re^{I}$ is an essential extension of $\phi^{\prime}\left(\mathfrak{A}^{\prime}\right)$. Hence $I_{2}=\bigcup_{i \in I_{0}} J(i)$ and similarly $I_{3}=\bigcup_{i \in I_{1}} J^{\prime}(i)$. Now suppose that $j, k \in J(i)$ with $j \neq k$. Then for any $f \in A^{\prime}, \phi(f)(j)=\phi(f)(k)$. Once again $\left.\theta_{j}\right|_{\phi\left(A^{\prime}\right)}=\omega$ contradicting the assumption that $\S^{I}$ is an essential extension of $\phi\left(\mathscr{Y}{ }^{\prime}\right)$. Thus $|J(i)|=1$ for $i \in I_{0}$ and similarly $\left|J^{\prime}(i)\right|=1$ for $i \in I_{1}$.

Therefore $\phi_{0}$ is a bijection from $I_{0}$ to $I_{2} ; \phi_{1}$ is a bijection from $I_{1}$ to $I_{3}$, and $\phi(f)\left(\phi_{0}(i)\right)=f(i)$ for any $i \in I_{0}$ while $\phi(f)\left(\phi_{1}(i)\right)=f(i)$ for any $i \in I_{1}$. Thus $2^{\prime} \in \operatorname{Amal}(\mathcal{K}(\Re))$ as claimed. This indicates that any characterization of Amal $(K(\Re))$ which does not refer to extensions of homorphisms will likely be very complicated.

Problem. Is $(* *)$ necessary?

10. Monadic algebras. An algebra $\mathscr{U}=\langle A ; \Lambda, \vee-, 0,1, C\rangle$ is a monadic algebra if $\langle A ; \Lambda, \vee,-, 0,1\rangle$ is a Boolean algebra and $C$ is a closure operator (i.e. $C C a=C a \geq a, C 0=0, C(a \vee b)=C a \vee C b)$ satisfying $C(C a \wedge b)=C a \wedge C b$. Monadic algebras were introduced by Halmos in his study of algebraic logic [0]. All subdirectly irreducible monadic algebras are simple and $\mathcal{Q}$ is simple iff $\mathrm{Ca}=$ 1 for every nonzero $a \in A$. For $1 \leq n<\infty$ let $\mathcal{U}_{n}$ be a simple monadic algebra with $n$ atoms. Every finite nontrivial simple monadic algebra is isomorphic to 
some $\mathscr{U}_{i}$ and every subalgebra of $\mathscr{U}_{i}$ is isomorphic to some $\mathscr{U}_{j}$ for $1 \leq j \leq i$. Since monadic algebras have Boolean algebras as reducts, they have distributive and permutable congruences. Thus each $\mathscr{U}_{i}$ is quasi-primal. The ternary discriminator function can be represented on each $\mathcal{X}_{i}$ by $t(x, y, z)=(C(x+y) \wedge x) \vee$ $(-C(x+y) \wedge z)$ where $x+y$ is the symmetric difference of $x$ and $y$. (In fact, $t(x, y, z)$ represents the ternary discriminator function on every simple monadic algebra.) Let $K_{i}$ be the equational class generated by $\mathscr{N}_{i}$; let $K_{0}$ be the class of trivial monadic algebras, and $K_{\infty}$ the class of all monadic algebras. In [11] Monk shows that the lattice of equational classes of monadic algebras is $K_{0} \subset$ $K_{1} \subset \ldots \subset K_{\alpha^{\prime}}$

Bass has shown that monadic algebras are locally finite (i.e. every finitely generated algebra is finite) and has calculated the free monadic algebra on $n$ generators (see [2]). Note that a simple monadic algebra is $n$-generated iff it is $n$-generated as a Boolean algebra. Thus every finitely generated subdirectly irreducible monadic algebra is finite and for each $n$ there are only finitely many $n$-generated subdirectly irreducibles. This is equivalent to local finiteness.

Let $\mathcal{F}(m)$ be the free algebra in $\mathcal{K}_{\infty}$ on $m$ free generators and $\mathcal{F}_{n}(m)$ the free algebra in $\mathcal{K}_{n}$ on $m$ free generators. Note that $\mathcal{F}(m) \simeq \mathcal{F}_{2} m(m)$. We know from Theorem 2.2 that $\mathcal{F}(m) \simeq \Pi_{i=1}^{2 m} \mathfrak{r l}_{i}^{\alpha(i, m)}$ where $\alpha(i, m)$ is the number of dual atoms of $\mathcal{C}(\mathcal{F}(m))$ which send $\mathcal{F}(m)$ onto $\mathscr{N}_{i}$. To calculate $a(i, m)$ note that $\mathcal{F}(m)$ contains $\mathscr{B}(m)$, the free Boolean algebra on $m$ generators, as a Boolean subalgebra (i.e. the $m$-ary Boolean polynomials). Let $\phi: \mathcal{F}(m) \rightarrow \mathcal{F}(m) / \theta \simeq \mathfrak{U}_{i}$ be the canonical homomorphism. Then $\phi$ sends $B(m)$ onto $\mathcal{F}(m) / \theta$ and $\phi$ is determined by its restriction to $B(m)$ (since $C a$ is $\{0,1\}$-valued in $\mathscr{Q}_{i}$ ). Exactly $2^{m}-i$ atoms of $B(m)$ are mapped onto 0 by $\phi$, and once these $2^{m}-i$ atoms are determined, $\theta$ is uniquely determined. Thus

$$
a(i, m)=\left(\begin{array}{c}
2^{m} \\
i
\end{array}\right)
$$

For $i=1$ or 2 every $\phi \in \mathscr{I}\left(\mathscr{U}_{i}\right)$ can be extended to an automorphism of $\mathscr{U}_{i}$ while for $i \geq 3$ this is not true. Thus by Theorem 2.3 the injectives in $K_{i}$ for $i=1$ or 2 are the extensions of $\mathscr{U}_{i}$ by complete Boolean algebras; for $3 \leq i<\infty$ there are no nontrivial injectives in $K_{i}$. That $K_{\infty}$ has no nontrivial injectives follows from the facts that $K_{\infty}$ has simple algebras of arbitrarily large cardinality and that every nontrivial monadic algebra has a subalgebra isomorphic to $\mathscr{U}_{1}$. In $K_{1}, C a=a$ so that the injectives in $K_{1}$ are just those algebras in $K_{1}$ which are complete as Boolean algebras. A similar result holds in $K_{2}$ : in $K_{2}$ the injectives are the Boolean extensions of $\mathscr{O}_{2}$ which are complete as Boolean algebras. The proof of this is a straightforward but tedious calculation. 


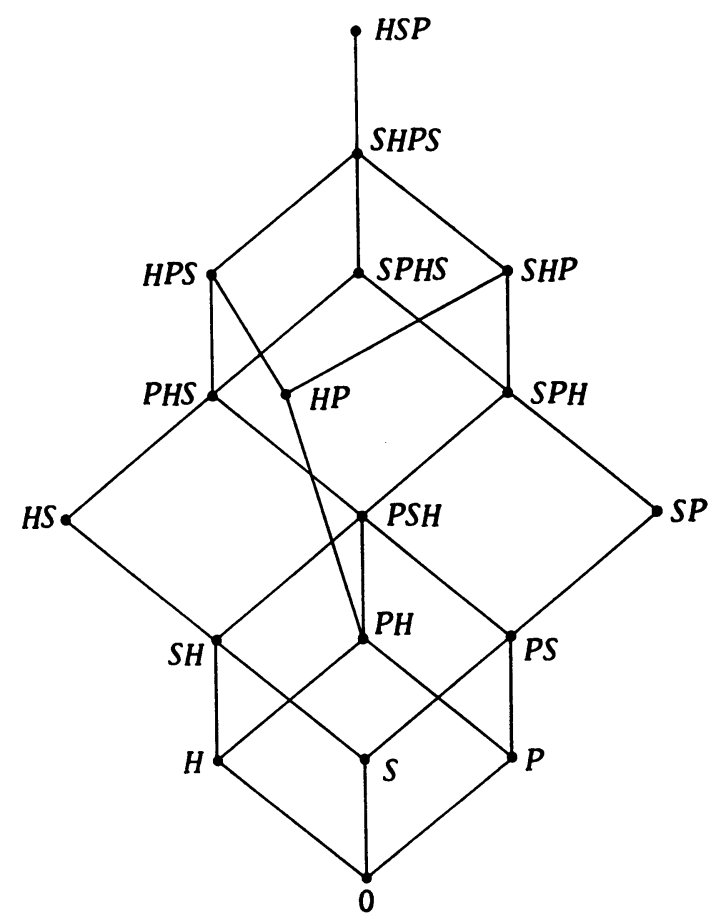

Figure 1

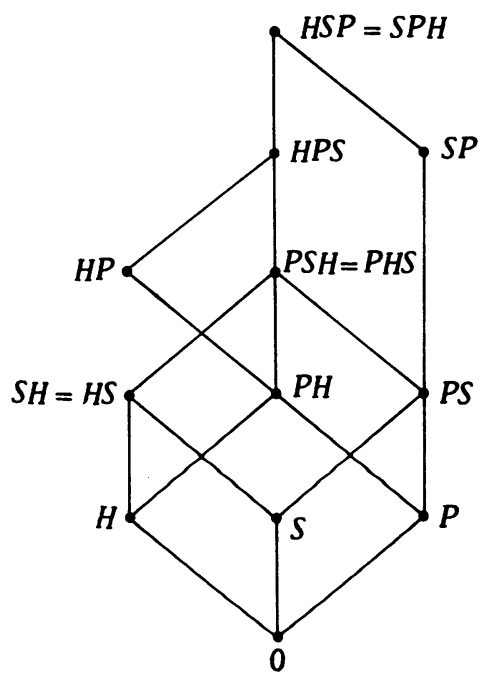

Figure 2 


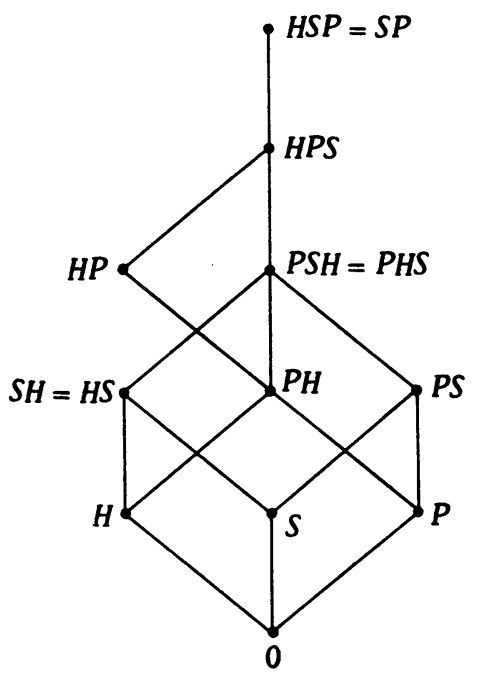

Figure 3

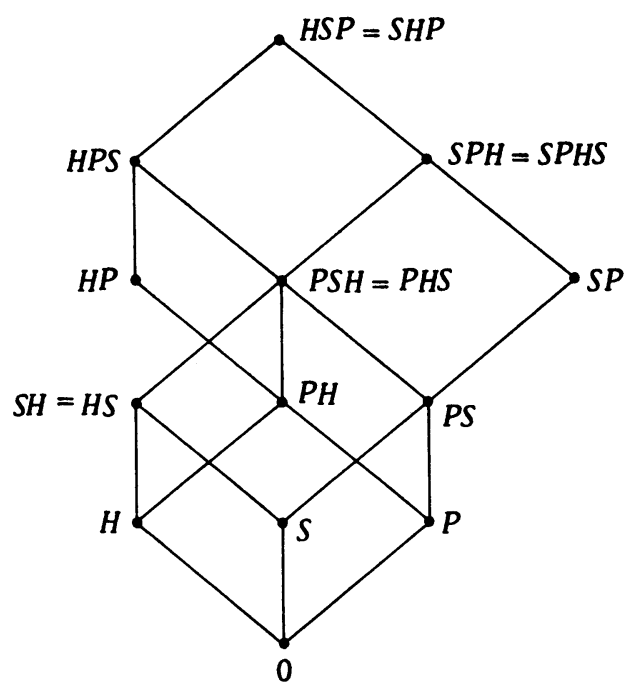

Figure 4 
By Theorem 3.5 $K_{n}$ has the C.E.P. for $1 \leq n<\infty$. To see that $K_{\infty}$ has the C.E.P. note that to prove Theorem 3.2 we need only assume that there is a fixed polynomial which evaluates to $n(x, y, u, v)$ on each subdirectly irreducible in the equational class. The polynomial $(C(x+y) \wedge v) \vee(-C(x+y) \wedge u)$ works in $K_{\infty}$.

By Theorem 5.4 the standard semigroup for $K_{1}$ is given by Figure 3; by Theorem 5.3 the standard semigroup for $K_{n}$ with $2 \leq n<\infty$ is given by Figure 2. To calculate the standard semigroup for $K_{\infty}^{n}$ first note that since $K_{n} \subset K_{\infty}$, Figure 3 is a homomorphic image of the standard semigroup for $K_{\infty} \cdot$ Next, $H S=S H$ in $K_{\infty}$ since $K_{\infty}$ has the C.E.P. Finally, $H S P \neq S P H$ in $K_{\infty}$ since if $\mathscr{V}_{1}, \mathscr{U}_{2}$ are infinite simple monadic algebras with $\left|A_{1}\right|<\left|A_{2}\right|$ then $\mathscr{U}_{2} \notin S P H\left(\mathscr{U}_{1}\right)$ while $H S P\left(\mathscr{U}_{1}\right)$ $=K_{\infty^{\circ}}$. Thus the standard semigroup for $K_{\infty}$ is given by Figure 4 .

It is clear from Theorem 6.2 that $K_{1}$ and $K_{2}$ have the A.P. but that, for $3 \leq$ $n<\infty, K_{n}$ does not. To prove that $K_{\infty}$ has the A.P. we will use Corollary 6.4. Thus we have to amalgamate $\langle\mathscr{U} ; \beta, \mathscr{B}, \gamma, \mathfrak{C}\rangle$ where $\mathscr{U}, \mathfrak{D}, \mathcal{E}$ are simple monadic algebras and $\mathscr{U}$ is nontrivial. Since Boolean algebras have the A.P. we can find a Boolean algebra $\mathfrak{D}$ which is an amalgam of $\langle\mathscr{H} ; \beta, \mathscr{B}, \gamma, \mathfrak{C}\rangle$ as Boolean algebras. Turn $\mathfrak{D}$ into a simple monadic algebra and we have done the amalgamation in $K_{\infty}$ :

By Theorem 7.5 the weak injectives in $K_{n}$ are the extensions of $\mathscr{U}_{n}$ by complete Boolean algebras. More interesting is the question of what, if any, are the weak injectives in $K_{\alpha^{\prime}}$. Let $\mathcal{U}^{\prime}$ be a weak injective in $K_{\alpha^{\circ}}$. Then there is a simple monadic algebra $\mathscr{U}$ such that $\mathscr{U}$ ' is embeddable in $\mathscr{U}^{I}$ for some $I$ and $|A| \geq$ $\mid A^{\prime} \%$. Since $\mathcal{U}^{\prime}$ is a weak injective, there is a homomorphism $\phi$ from $\mathfrak{U}^{I}$ onto $\mathcal{U}^{\prime}$. But $\mathcal{U}$ is simple and $|A| \geq\left|A^{\prime}\right|$ so restricting $\phi$ to the diagonal subalgebra of $\mathfrak{U}^{l}$ means that $\mathfrak{U}^{\prime}$ must be trivial. Thus $K_{\infty}$ has no nontrivial weak injectives.

It must be pointed out that much of the material in this section for which no reference is given is known although it may not have been published previously.

\section{REFERENCES}

1. B. Banaschewski, Injectivity and essential extensions in equational classes of algebras, Proc. Conf. on Universal Algebra (Queen's Univ., Kingston, Ont., 1969), Queen's Univ., Kingston, Ont., 1970, pp. 131-147. MR 41 \#3354.

2. H. Bass, Finite monadic algebras, Proc. Amer. Math. Soc. 9 (1958), 258-268. MR $20 \# 827$.

3. S. D. Comer and J. S. Johnson, The standard semigroup of operators of a variety, Algebra Universalis 2 (1972), 77-79. MR 45 \$8740.

4. A. Day, A note on the congruence extension property, Algebra Universalis 1 (1971), 234-235.

5. A. Foster, Generalized "Boolean" theory of universal algebras, Part I: Subdirect sums and normal representation theorem, Math. Z. 58 (1953), 306-336. MR 15, 194.

6. G. Gratzer, Universal algebra, Van Nostrand, Princeton, N. J., 1968. 
7. G. Grätzer and H. Lakser, The structure of pseudocomplemented distributive lattices. II: Congruence extension and amalgamation, Tran s. Amer. Math. Soc. 156 (1971), 343-358. MR 43 \#124.

8. - The structure of pseudocomplemented distributive lattices. III: Injectives and absolute subretracts, Trans. Amer. Mach. Soc. 169 (1972), 475-488.

9. P. Halmos, Algebraic logic, Chelsea, New York, 1962. MR 24 \#A1808.

10. T.-K. Hu, On the topological duality for primal algebra theory, Algebra Universalis 1 (1971), 152-154.

11. D. Monk, On equational classes of algebraic versions of logic. I, Math. Scand. 27 (1970), 53-71. MR 43 \#6065.

12. D. Pigozzi, On some operations on classes of algebras, Notices Amer. Math. Soc. 13 (1966), 829 (Abstract \#639-1).

13. A. Pixley, Functionally complete algebras generating distributive and permutable classes, Math. Z. 114 (1970), 361-372. MR 41 \#6758.

14. - The ternary discriminator function in universal algebra, Math. Ann. 191 (1971), 167-180. MR 45 \#1820.

15. R. Quacken bush, Demi-semi-primal algebras and Mal'cev type conditions, Math. Z. 122 (1971), 166-176.

DEPARTMENT OF MATHEMATICS, UNIVERSITY OF MANITOBA, WINNIPEG, MANITOBA, CANADA 